\title{
FAILURE MECHANISMS IN WET TANTALUM CAPACITORS
}

\author{
D. HAYWARD \\ The Plessey Company Limited, Northants, U.K.
}

(Received May 13, 1975, in final form June 17, 1975)

\begin{abstract}
The wet tantalum capacitor is well established in both power supply smoothing and timing applications and has a well proven high reliability. This paper reviews the mechanisms of the possible failures arising from mis-application or over-stressing. The effect of over voltage on the anodic oxide is considered and the influence of cathode structure on reverse voltage and ripple current capability is discussed. The silver cathode allows migration of silver ions through the electrolyte under appropriate conditions and hence produces a failure mechanism not present in the tantalum cathode device. The life of a wet tantalum unit is eventually limited by loss of water vapour leading to open-circuit. Results are presented for button style capacitors which suggest that this is in fact not a practical limitation.
\end{abstract}

\section{INTRODUCTION}

In these days of advanced integrated circuitry, it is easy to overlook the intricacies of an apparently simple component such as a capacitor. Electrolytic capacitors in particular have a technology of their own and it is useful to understand the basic principles involved. The wet tantalum device is used primarily in the military, avionic and professional equipment industry where its high reliability ${ }^{1}$ and long working life are essential. Most of the small numbers of failures that do occur can be attributed to misuse. Thus, although the onus must lie with the manufacturer to specify his product capability, some background knowledge will help the circuit designer to avoid the more obvious pit falls.

The detailed construction of wet tantalum capacitors is adequately covered elsewhere ${ }^{2}$ but brief mention of a few features will assist in the following discussion.

The anode is manufactured by pressing high purity tantalum powder and sintering into a porous slug. The purity of the starting material and the degree of purification achieved during the vacuum sintering operation control to a large extent the leakage current and reliability of the final device. The tantalum pentoxide dielectric is formed over the total slug surface by anodic oxidation, normally at constant current and elevated temperature, in a suitable aqueous or partially açueous electrolyte.

The counter electrode in the unit is the working electrolyte, most commonly sulphuric acid, and the whole is assembled in a suitable case. The two main types of construction are shown in Figure 1. In both instances a high capacitance is required at the electrolyte to case interface in order that the capacitance of the overall device is controlled essentially by the anode film. For the silver case this is usually achieved by applying a black platinum layer over the lightly etched silver surface. In the tantalum cased device, a porous tantalum "cathode", similar in construction to the anode, is sintered into the case. A thin, low voltage dielectric is anodically formed to give the required high capacitance.

Silver is chosen as a case material because of its good chemical resistance to conventional working electrolytes and its suitability for drawing into a can and forming a good spun seal. Tantalum has the advantage of resisting electrochemical dissolution in the working electrolyte as is discussed in this paper.

\section{ELECTRICAL FAILURE MECHANISMS}

\subsection{Over Voltage}

The dielectric is formed by anodisation at a voltage of at least $140 \%$ of the rated working voltage of the device. This gives a high safety margin and contributes towards the characteristic low leakage current of the wet tantalum capacitor.

The capacitor working electrolyte itself is a very suitable anodising medium and so the application of a voltage across the capacitor can itself result in anodisation. Thus any minor damage or imperfections 


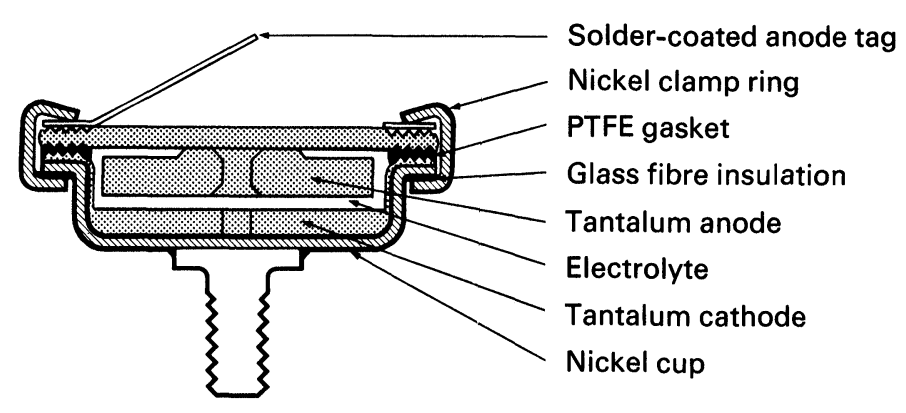

(a)

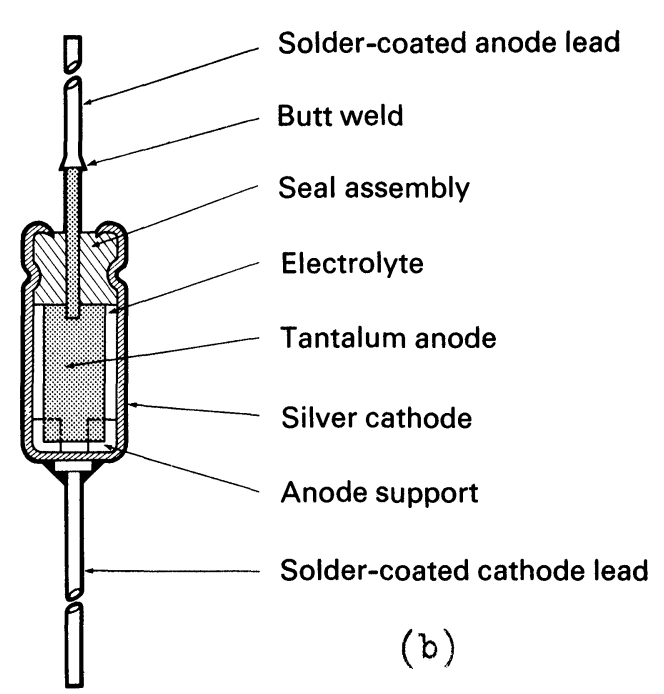

FIGURE 1 Typical capacitor constructions: (a) button style; (b) tubular style.

can be healed in-situ. In fact the tantalum oxide dielectric is extremely stable in the working electrolyte and in contrast to most aluminium electrolytics no general "reforming" of the dielectric is required, even after extended periods on shelf.

To produce any general thickening of the dielectric by anodisation it is, in theory, necessary to exceed the original forming voltage. In fact the rate of film growth at constant voltage decays exponentially and the limiting thickness is not reached during manufacture. Thus, in practice some growth of the film may occur, over a period of time, at voltages below the original forming voltage. This type of consolidation of the dielectric normally causes a fall in leakage current with time of application of voltage e.g. on endurance testing.

Thus the effect of over-voltage, defined as application of a voltage in excess of the rated working value, on a capacitor can be explained. As the leakage current characteristic (Figure 2.) indicates, the effect of small over-voltage is to increase the leakage current, but this may well decrease with time. As the original anodising stress is approached and then exceeded the current increases more rapidly as current is now used in increasing the dielectric thickness with a consequent decrease in capacitance.

Normally any thickening of the oxide will give a capacitance change of not more than a few percent. A useful diagnostic tool in such a case is the change in anode colour. Each thickness of dielectric has a characteristic colour derived from interference be- tween light reflected from the tantalum - tantalum oxide interface and the tantalum oxide-air interface. This colour is very sensitive to minor variations in dielectric thickness and hence over-voltage of sufficient magnitude to cause even small changes can be diagnosed by opening the capacitor.

If the voltage is raised further a level is reached at which the anodic oxide - electrolyte interface be-

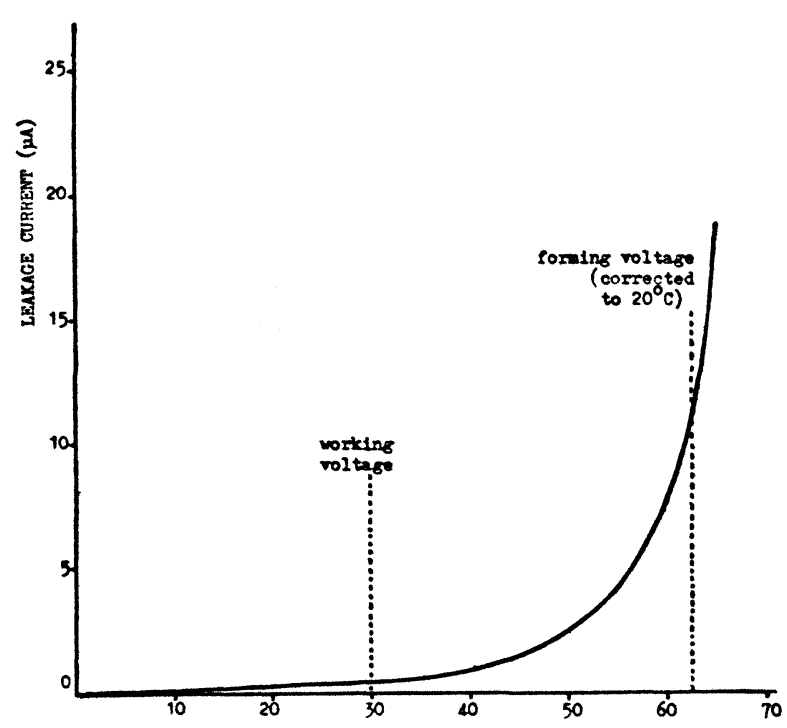

FIGURE 2 Leakage current v. applied voltage at room temperature for a $140 \mu \mathrm{F} / 30 \mathrm{~V}$ capacitor. 
comes unstable and local breakdown may occur. If this breakdown is limited, as in the case caused by a short duration high voltage spike, the dielectric may effectively heal. If the potential is maintained a condition known as scintillation develops, in which a spark discharge runs across the oxide surface causing thermal oxidation. This punctures the anodic oxide and results in high leakage and eventually a short circuit. The breakdown voltage depends on the electrolyte, the original dielectric thickness, the temperature and the available current density. Table I shows typical values, for two capacitor ratings, ob-

TABLE I

Capacitor breakdown voltages.

\begin{tabular}{|c|c|c|}
\hline Rating & $\begin{array}{l}\text { Breakdown } \\
\text { at } 20 \mathrm{C}\end{array}$ & $\begin{array}{l}\text { Breakdown } \\
\text { at } 125^{\circ} \mathrm{C}\end{array}$ \\
\hline $\begin{array}{r}140 \mathrm{uF} / 30 \mathrm{~V} \\
47 \mu \mathrm{F} / 75 \mathrm{~V}\end{array}$ & $\begin{array}{c}84 \mathrm{~V}(68 \mathrm{~V}) \\
149 \mathrm{~V}(188 \mathrm{~V})\end{array}$ & $\begin{array}{c}64 \mathrm{~V}(51 \mathrm{~V}) \\
142 \mathrm{~V}(135 \mathrm{~V})\end{array}$ \\
\hline
\end{tabular}

tained by increasing the voltage at approximately $2 \mathrm{~V} / \mathrm{s}$ with a current limitation of $2 \mathrm{~mA}$.

The corresponding forming voltages, corrected to the relevant temperatures are included in parentheses. Thus the $75 \mathrm{~V}$ device scintillated at $20^{\circ} \mathrm{C}$ before the voltage reached the forming level, while in the other cases some additional anodisation is obviously possible before breakdown.

It should not be assumed that voltages between the forming and breakdown values can necessarily be withstood for an appreciable length of time, since increased leakage current may cuase local heating and promote breakdown. If the current through the capacitor becomes high, as for example after scintillation, internal heating and hydrogen gas evolved at the cathode will tend to pressurise the device and this may precipitate seal failure as will be discussed below.

Thus, although the designer may feel that there is little danger in exceeding the d.c. rated working voltage provided the level of leakage current remains acceptable and scintillation is not reached, increased stress above the working level recommended is likely to cause a reduction in reliability. Hence, although frequent surges up to $115 \%$ of the rated value are quite permissible it is wise to regard the rated continuous voltage as law and maintain a good safety margin.

In this context one other feature is worth mentioning - field crystallisation. The anodic oxide is nor- mally amorphous in structure but under conditions of high voltage and temperature this may, in appropriate electrolytes, be replaced by a crystalline oxide radiating from impurity centres ${ }^{3}$. The current used in growing this new crystalline oxide gives an apparent increase in leakage current. The crystalline oxide is a poorer dielectric and the breakdown voltage is reduced. This effect is virtually unknown in wet tantalum units operated within their rated capability since the sintering operation greatly reduces the level of surface impurity of the anode. However, this phenomenon can become important if units are held for long periods at high temperature near the initial forming voltage and manifests itself in increased leakage current which may "run away" causing heating and eventual breakdown.

\subsection{Reverse Voltage}

The tantalum/tantalum oxide/wet electrolyte system exhibits rectifying properties as shown in Figure 3 and the current flow in the reverse direction will be comparatively large for even low voltages. There is a complication arising from the role of the cathode. In a silver cased unit, reverse voltage places the silver at a positive potential relative to the electrolyte and silver ions will then pass into solution. This will disrupt the cathode surface and if a black platinum layer has

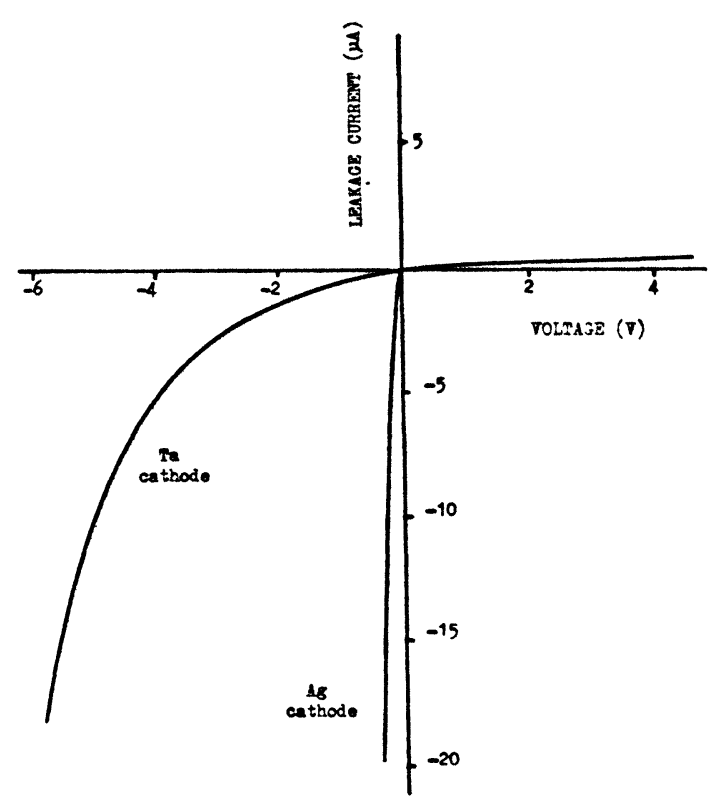

FIGURE 3 Reverse leakage current characteristics. 
been applied to increase the cathode surface area this too may be stripped. If the reverse bias is maintained, silver will migrate to the anode (now negatively biased) and may deposit onto the dielectric. A variety of explanations have been advanced to explain the rectifying nature of the oxide/electrolyte interface 4 but undoubtedly a large proportion of the reverse current flows through imperfections in the oxide. It is at these sites that silver is predominantly deposited. At low reverse potentials, and hence lower current density, dendritic deposits are obtained. At higher levels deposition is more general. The presence of silver on the anode surface maintians a directly conducting path through the dielectric which produces increased leakage current and if appreciable deposits are allowed to build up this may again result in early failure. In those devices having tantalum cathodes, the cathode itself has been provided with its own thin anodic film. When the capacitor as a whole is reverse biased, this cathode dielectric will in fact be forward biased so that current flow is still restricted (see Figure 3 ). The cathode is normally formed to give protection up to $3 \mathrm{~V}$ in reverse. Application of a reverse voltage beyond this level gives increased current which may simply thicken the cathode dielectric, or cause thermal run-away. The $3 \mathrm{~V}$ protection offered here is ample to cover the requirements of the majority of those circuits where occasional excursions through zero bias can occur.

\subsection{Ripple Current}

The maximum ripple current which can safely be passed by a tantalum capacitor is one of the most difficult parameters to specify. Obviously, the applied ripple voltage must not, when added to the d.c. voltage, either overvolt or reverse volt the device. The maximum ripple rating must also be related to the maximum temperature within the device consistent with satisfactory performance. Ambient temperature is thus a critical factor, with the temperature rise resulting from the passage of ripple current depending on the capacitor equivalent series resistance. Of equal importance is that the temperature will depend on the prevailing cooling conditions and the degree of heat sinking. This is illustrated in Figure 4. Varying ripple currents were passed through a button capacitor mounted in still air at room temperature, until thermal equilibrium was reached. The case temperature was measured by means of a thermocouple clamped to the anode plate. Results were recorded with the capacitor mounted either on a thermally insulating block, on a power heat sink or with the capacitor encapsulated in silicone rubber to reduce cooling effects.

A further complication arises because the dissipative element itself, the capacitor equivalent series resistance (e.s.r.) decreases with temperature and also frequency. In the face of such a complex situation

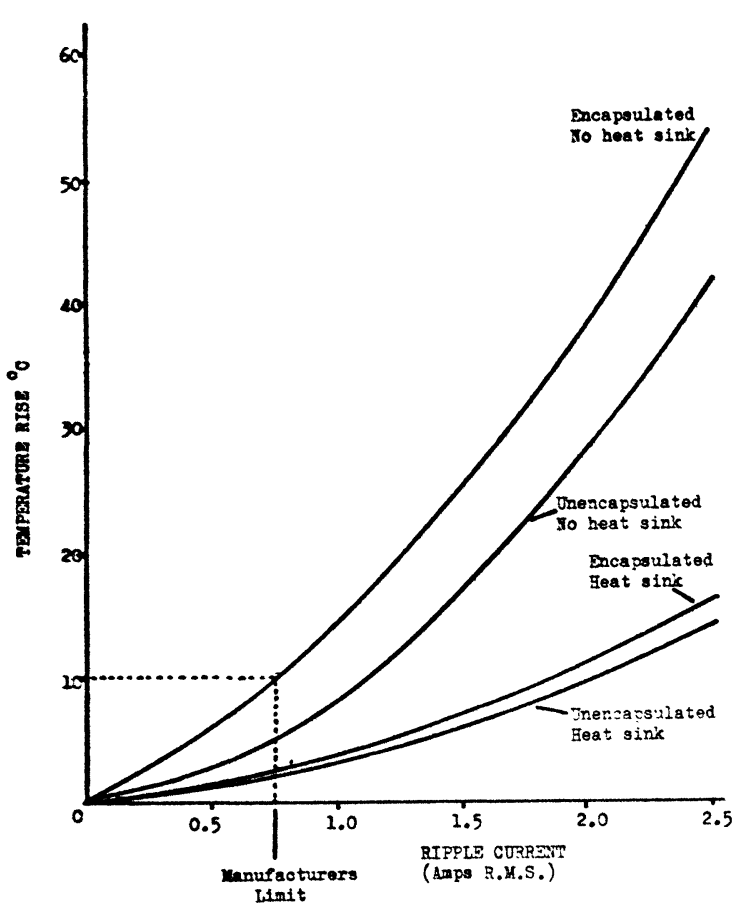

FIGURE 4 Temperature rise v. ripple current for a variety of cooling conditions.

the manufacturer normally specifies for worst case conditions in terms of allowable r.m.s. current. In the case illustrated the manufacturers limit corresponds to a worst case temperature rise of $5^{\circ} \mathrm{C}$. Thus it is possible that some extension of a.c. loading is permissable in appropriate conditions although as the above suggests the user is well advised to consult the manufacturer.

Over stressing with regard to ripple level thus leads to an increase in capacitor temperature and hence the failure mechanism may be dealt with under temperature effects. (See section 3.1.)

The above theory may be applied to the tantalum cathode series of units. However, with the silver cased style a second feature is present which normally over-rides the simple heating effect. 
It has already been mentioned that a silver cathode can interact with the electrolyte when reverse voltage is applied. Let us consider what will occur to a unit biased in the forward direction with a superimposed a.c. signal. On the charging cycle the case is cathodic and on the discharge cycle it is anodic relative to its rest value. Hence silver ions can enter the electrolyte during a discharge phase. Provided these silver ions have not diffused too far from the case surface, they will be replated onto the cathode during a subsequent charging cycle. Thus, for a symmetrical high frequency waveform and with insignificant ion diffusion, no appreciable build up of silver ion will result provided the amplitude is limited. The cathode surface may be somewhat disrupted but is unlikely to cause any noticeable change in overall capacitance. If the current flow is high a large local accumulation of silver ions will occur and with the usual sulphuric acid electrolyte, silver sulphate may even precipitate, ${ }^{5}$ and any such deposits will tend to keep the electrolyte generally saturated in silver ion. In addition, if the waveform is unsymmetrical, dissolution and plating of silver at the cathode will not be completely reversible and with suitable waveforms excess silver may again accumulate in the electrolyte. The tantalum anode will also by cycling about its rest potential and if silver ions diffuse to the surface of the anode they may deposit there during a discharge cycle. Any build up of silver on the anode brings the risk of increased leakage current and eventually a short circuit. The presence of the local cell formed between the silver case and the black platinum indicates that there is always a small amount of silver in solution, even on forward bias, though not in a sufficient quantity to cause any deleterious effects.

This phenomenon has been recently studied in tests carried out at the Plessey Product Assessment Laboratories. ${ }^{6}$ The matrix shown in Table II was set up.

There were ten silver cased tubular style capacitors in each cell, and these were run for $8,000 \mathrm{~h}$ at $85^{\circ} \mathrm{C}$, five with the manufacturer's rated ripple current of $180 \mathrm{~mA}$ r.m.s. and five at twice this current. An appropriate d.c. bias was applied to prevent reverse volt excursions.

Capacitors were measured before and after test and representative samples were opened for internal examination. Average capacitance changes in each cell of the matrix were small and all leakage currents remained below $1 \mu \mathrm{A}$. Silver deposits were found on the anodes and cathodes of capacitors from all groups. As expected more silver was deposited in those capacitors run at the higher ripple current amplitude.
The results also suggest that for a given wave shape and amplitude the amount of deposit increases with decreasing frequency. Thus with the sine wave at $50 \mathrm{~Hz}$ a large build up of silver was found on the anode and large areas of the cathode were covered with redeposited silver. The units were, in the cases where $360 \mathrm{~mA}$ was applied, close to silver bridge formation between anode and cathode. At $400 \mathrm{~Hz}$ isolated lumps of silver were found on the anode, with silver tracks on the cathode. At $2.5 \mathrm{kHz}$ small amounts of silver were present on the anode, either as a thin layer or in isolated sites, and only small tracks of redeposited silver were visable on the cathode. At $20 \mathrm{kHz}$ the position was similar but again the degree of deposition was reduced. As already indicated this effect is probably related to ion migration. At low frequency the charge transferred per cycle is greater, as also is the time during which silver ions can diffuse. Hence at the lower frequency levels both more dissolution and more migration, with subsequent redeposition on the anode, can occur.

TABLE II

Matrix showing ripple testing criteria applied to silver-cased tantalum capacitors.

\begin{tabular}{lllll}
\hline & \multicolumn{4}{c}{ Frequency (Hz) } \\
\cline { 2 - 5 } Voltage waveform & 50 & 400 & $2.5 \mathrm{k}$ & $20 \mathrm{k}$ \\
\hline Sine wave & $\sqrt{ }$ & $\sqrt{ }$ & $\sqrt{ }$ & $\sqrt{ }$ \\
$\begin{array}{l}\text { Ramp } \\
\text { Square } \\
\text { Inverter supply }\end{array}$ & $\sqrt{ }$ & $\sqrt{ }$ & $\sqrt{ }$ & \\
\hline
\end{tabular}

With the exception of the $50 \mathrm{~Hz}$ samples relatively little difference in levels of deposit were observed with differing waveform. At $50 \mathrm{~Hz}$ the sine wave gave more deposition than the ramp wave form. The ramp consists of a relatively quick discharge during which silver may dissolve. Before appreciable diffusion can occur the relatively long charge begins and the silver is replated. Thus little silver will reach the anode even at comparatively low frequencies, but will be evenly deposited back at the cathode. Even, so the difference in levels of deposit found on the cathodes at $50 \mathrm{~Hz}$ was surprising. A harmonic analysis shows that at $50 \mathrm{~Hz}$ for the ramp waveform used, only $28 \%$ of the current was carried by the fundamental and this too must contribute to the levels of deposition observed. 


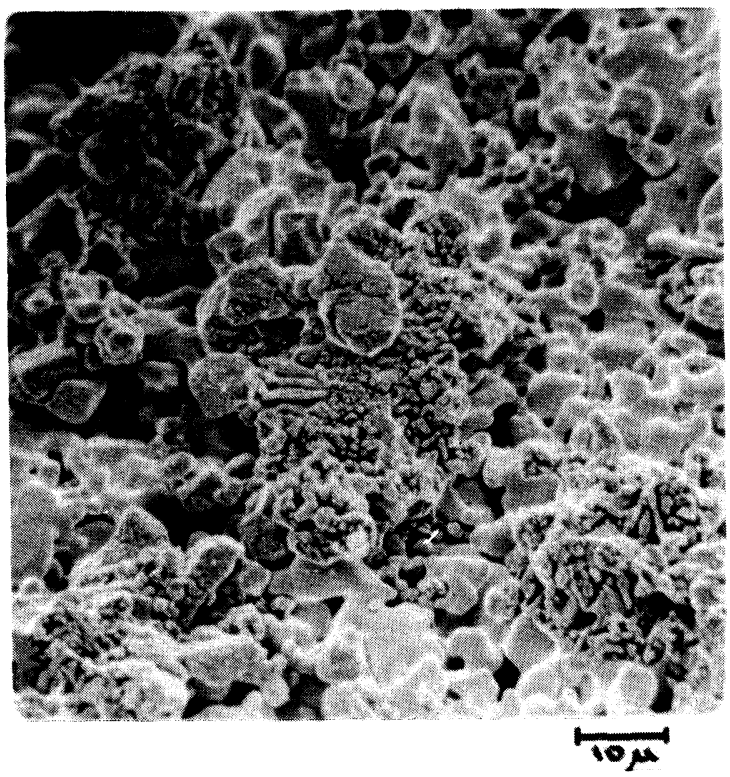

FIGURE 5 Silver deposit on an anode subjected to $50 \mathrm{~Hz}$ sine wave ripple testing at $85^{\circ} \mathrm{C}$ for $8,000 \mathrm{~h}$. Current level $180 \mathrm{~mA}$ r.m.s.

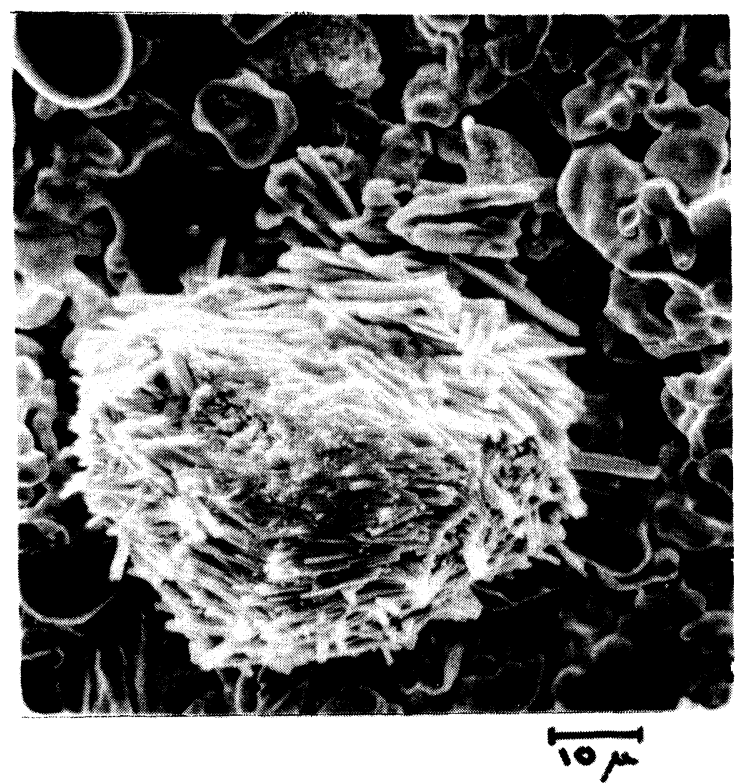

FIGURE 6 Silver deposit on an anode subjected to $20 \mathrm{kHz}$ sine wave ripple testing at $85^{\circ} \mathrm{C}$ for $8,000 \mathrm{~h}$. Current level $180 \mathrm{~mA}$ r.m.s.

The structure of the silver deposits also varies with conditions. On the anodes a granular structure was obtained at low frequencies, changing to a more dendritic deposit at higher frequencies. (Figures 5 and $6)$.
Since the trends observed are based in a limited number of test cells further work is underway to give a more detailed picture.

The above results suggest that the quoted ripple levels are well within the capacitor capability. It must be appreciated that the type of test conditions used were by no means worst case. In actual equipment the waveform is unlikely to remain stable over long periods and switch on and off pulses, noise, high frequencies, high amplitude transients etc. all complicate the picture. The degree of silver migration required to cause failure is also by no means constant and varies with individual units and on the structure of the deposit. The figures quoted as recommended maxima by most manufacturers have been derived from results both in the field and the laboratory.

With silver cathode units this migration effect is more important than internal heating and the high reliability experienced with these devices results from the setting of realistic ripple limits. The electrical failure mode associated with silver migration is high leakage current and eventually short circuit.

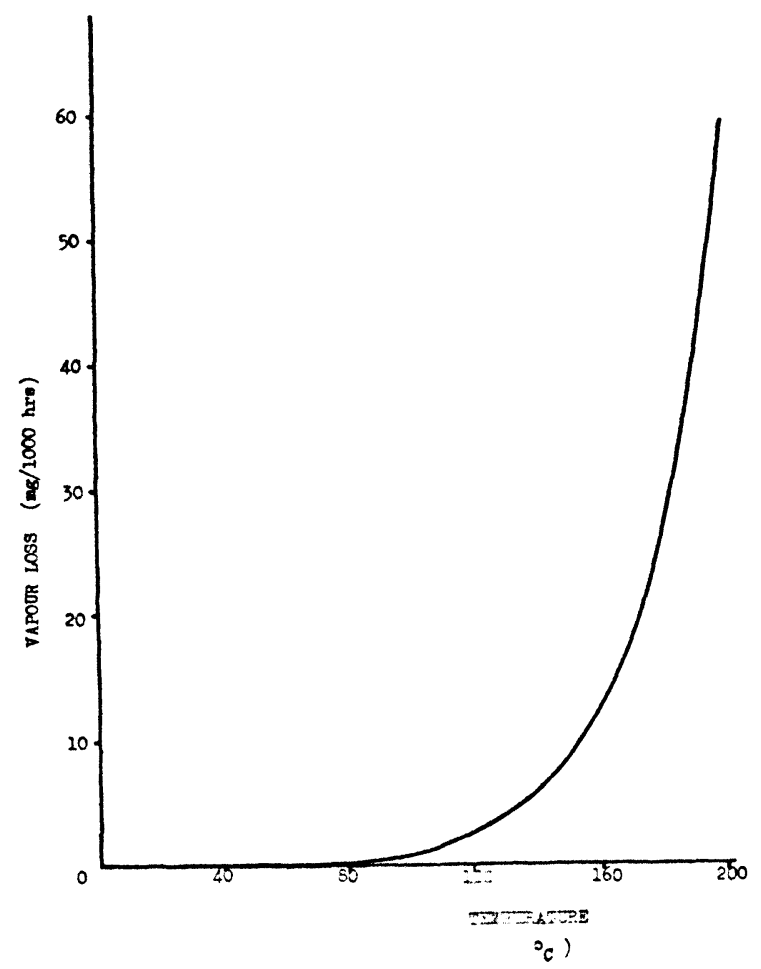

FIGURE 7 Loss of water vapour v. temperature for a button capacitor. 


\section{ENVIRONMENTAL FAILURE MECHANISMS}

\subsection{Temperature}

The main effects of an increase in temperature are increased leakage current and increased capacitance. Although neither of these are capacitor failures they may cause circuit failure if these parameters move outside the required tolerance. Increased temperature also aggrevates any of the previous effects which cause increased leakage current and speeds up runaway effects leading to breakdown.

At low temperature, the electrolyte used in devices rated to $-55^{\circ} \mathrm{C}$ freezes at $-64^{\circ} \mathrm{C}$ giving an opencircuit. However, as soon as the temperature is raised the electrolyte melts and works normally. Thus, indefinite storage at $-60^{\circ} \mathrm{C}$ is quite permissible.

Conventional seal materials such as p.t.f.e. and elastomeric materials have finite coefficients for diffusion of water vapour, and so the capacitor electrolyte will eventually dry out to produce an open circuit. The diffusion rate is greatly increased with increasing temperature and this is thus one of the factors controlling the maximum working temperature of the device. A plot of rate of loss of water vapour against temperature is shown in Figure 7 for a button style capacitor. This has been derived by maintaining a number of units at a variety of

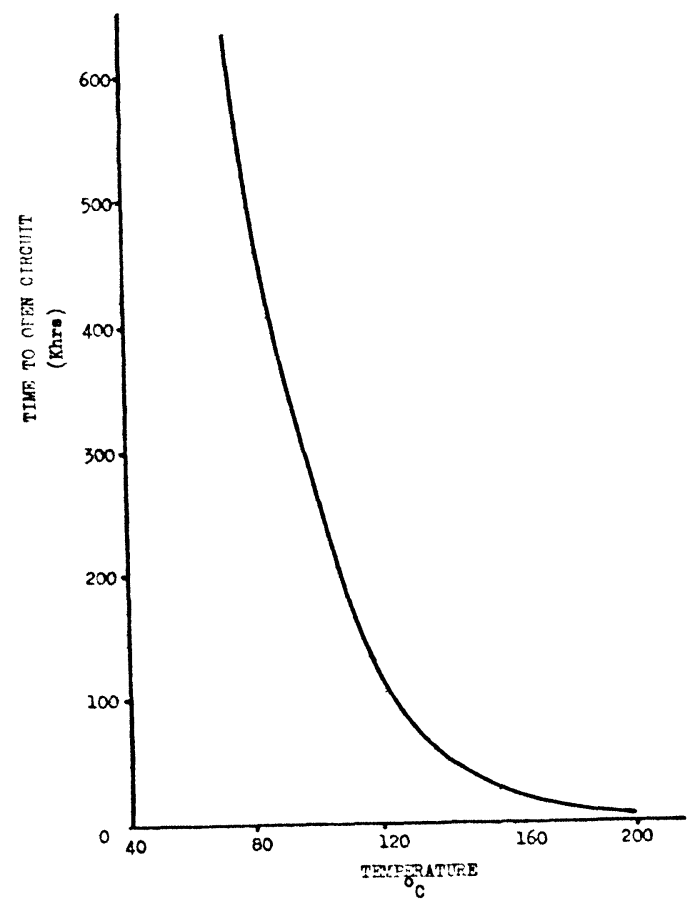

FIGURE 8 Time to open circuit against temperature. temperatures and periodically weighing. All units were given a $200^{\circ} \mathrm{C}$ pre-heat and the weight loss occuring due to loss of surface moisture etc. was ignored. A blank sample containing no electrolyte was also run at $200^{\circ} \mathrm{C}$ to confirm that weight loss was attributable to diffusion of water vapour.

If the loss of water vapour required to cause an open circuit is determined a "life-time" versus temperature plot can be constructed, for example Figure 8. Deterioration prior to open-circuit is limited to a gradual increase in dissipation factor. The actual position of the curve relative to the time axis will depend not only on the type of seal but also on the quantity of electrolyte in the device, its geometry and several other factors. Hence this should be taken as a typical illustration and should not be used to calculate life spans in the general case. Nevertheless, at $200^{\circ} \mathrm{C}$, a life time of the order of $5,000 \mathrm{~h}$ is predicted for this particular device. In practice capacitors are seldom held continuously at high temperatures and wear out from this mechanism is very rare outside the test laboratory. However, it is apparent that the lower the temperature the longer the anticipated life and hence cooling and heat sinking are again important considerations where high operational temperatures are likely.

There is a physical limit to the temperature that the capacitor will withstand. The electrolyte boils at $128^{\circ} \mathrm{C}$ at atmospheric pressure and hence considerable pressure develops at high temperature. However, with the present state of devices there is a considerable safety margin for both tubular units, normally rated up to $125^{\circ} \mathrm{C}$, and for button styles, rated up to $200^{\circ} \mathrm{C}$, before actual seal rupture will occur. The button style will, as its maximum temperature rating suggests, withstand rather higher internal pressures than the conventional tubular type.

Hermetic sealing is possible, but although this does reduce the vapour diffusion rate, it is doubtful if it is justified on this count alone. If pressurisation does occur within the unit as a result of mis-application, eventual release of the sealing may be more dramatic than the gradual easing of the seal in the conventional unit.

The dielectric itself is stable to temperatures in excess of $200^{\circ} \mathrm{C}$ although if no voltage is applied at temperatures above $150^{\circ} \mathrm{C}$ a small degree of oxygen migration may occur across the tantalum-tantalum oxide boundary. This increases the conductivity of the dielectric by creating free electrons on vacant anion sites. This causes a rise in capacitance ${ }^{7}$ which gradually decays when the voltage is re-applied and the vacancies are filled by re-anodisation. 
It is important to appreciate that a capacitor can heat up in a variety of ways. For example as well as high ambient temperature, excess ripple current, high leakage current caused by over-voltage etc. can result in high temperatures. Pressurisation can occur not only because of direct heating but also as a result of gas evolution within a capacitor as mentioned above.

\subsection{Radiation}

Relatively little has been published on the effects of radiation on tantalum capacitors. Although tantalum electrolytics have relatively low radiation thresholds they appear capable of withstanding extended exposure to low level radiation before suffering severe damage. Transient radiation is manifested as a sudden drop in the dielectric resistance of the capacitor but the device invariably recovers to its initial state. Thus the general effect of irradiation, whether u.v., $\gamma$-ray, $\mathrm{X}$-ray, or neutron bombardment, is an increase in leakage current which should return to its normal level after removal of the radiation.

Figure 9 shows a typical voltage/time plot for a capacitor subjected to a $75 \mathrm{~ns} \gamma$-ray pulse. The voltage loss is plotted in Figure 10 as a function of radiation dose. The total charge loss at a kilorad was between $0.6 \%$ and $2.1 \%$ over a range of capacitor values and is directly proportional to dosage. Values of e.s.r., capacitance and leakage current measured before and after irradiation indicated that there was no significant permanent effect. ${ }^{8}$

\subsection{Miscellaneous}

The wet tantalum series of capacitors, in particular

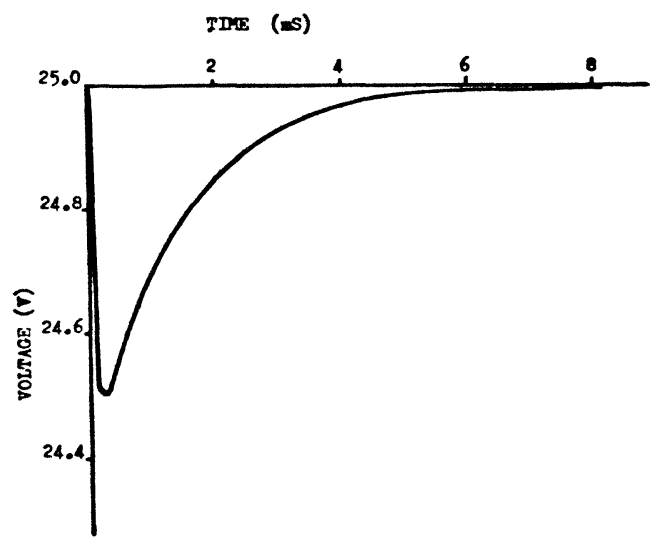

FIGURE $9 \quad 75 \mathrm{~V}$ capacitor subjected to a $75 \mathrm{~ns} 1280 \mathrm{rad}$ pulse. $25 \mathrm{~V}$ applied via a $10 \mathrm{k} \Omega$ series resistor. the button styles, are fairly robustly packed and mechanical failures are not common. The vibration, shock, bump and acceleration tests called up by the relevant $\mathrm{DEF}^{9}$ and B.S. ${ }^{10}$ specifications are severe enough to cover the majority of applications and the mechanical limitations of a circuit are most often defined by the capability of components other than the capacitor.

However, tubular units should not be fixed by the leads alone under conditions of high vibration as the stress may be sufficient to cause weakening of the lead wires. At vibration levels above the region of $5 \mathrm{~g}$ mounting should be by both body and leads.

In addition, tubular silver cased units do not withstand ultrasonic vibration for prolonged periods. Small amounts of platinum are detached from the cathode and some minor disruption of the oxide film may also occur although no permanent damage is caused.

Other tests such as resistance to humidity, salt mist, mould growth etc. are also normally considered in the specification for these devices. Failures are not normally encountered from these factors and any circuit designer with unusual severities in these directions is again best advised to consult the specification and/or the manufacturer.

\section{CONCLUSIONS}

The main failure modes have been reviewed. The wear-out mechanism of wet tantalum units is the loss of water vapour leading to open-circuit, although at rated temperatures, long life times are achieved. In

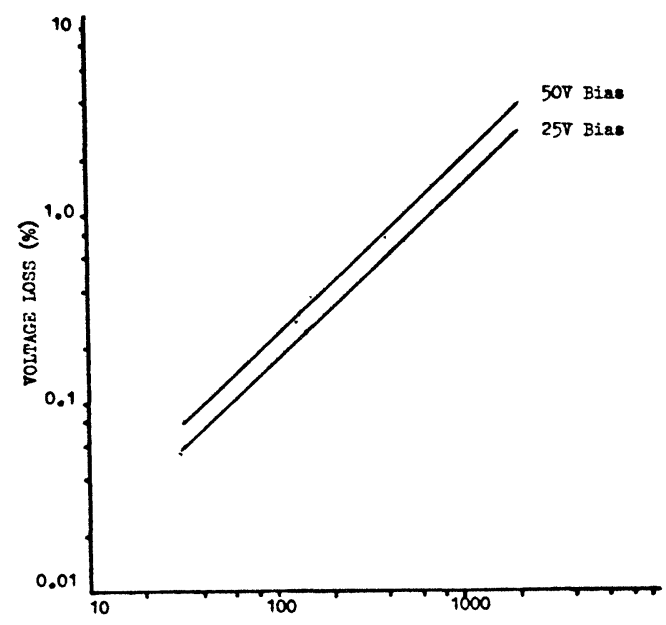

FIGURE 10 Voltage loss against radiation dose level for a 75 V capacitor. Pulse time 75 ns. 
cases where high ripple current or possible voltage reversals occur a silver cased device may suffer from migration of silver from the case to the anode with consequent failure. Hence, a tantalum cased device is recommended in such applications.

A well established high reliability should be anticipated for units operated within the rated temperatures and voltages. Use outside these limits is not completely eliminated as the manufacturer necessarily quotes for worst case conditions. However, because of the complexity of effects in the capacitor the circuit designer is well advised to consult the manufacturer on such occasions.

\section{ACKNOWLEDGEMENTS}

The author would like to thank MOD (PE) CVD, and Mr G. M. Matthews and Mr S. Wheatley of the Plessey Product
Assessment Laboratory for permission to use the experimental results on ripple current effects in silver cased units. This paper is published by permission of the Plessey Company Ltd.

\section{REFERENCES}

1. G. W. A. Dummer, Elec. Comp. Supp. Oct. 1970, p. 17.

2. J. M. Allen, New Electronics, July 1973, p. 84.

3. N. F. Jackson, J. Appl. Electron., 3, 91 (1973).

4. P. F. Schmidt, J. Electrochem. Soc., 115, 167 (1968).

5. W. J. Moore, A. W. H. Smith, Proc. 22nd Elec. Comp. Conf. 1972.

6. S. Wheatley, R.R. Britton, G. M. Matthews, Plessey Company Report No. 260012.

7. D. M. Smyth, G. A. Shirn, T. B. Tripp, J. Electrochem. Soc., 110, 1264, (1963).

8. R. Oakley, Private Communication.

9. DEF. STD. 59-21.

10. B.S. 9070 , Section 3 . 

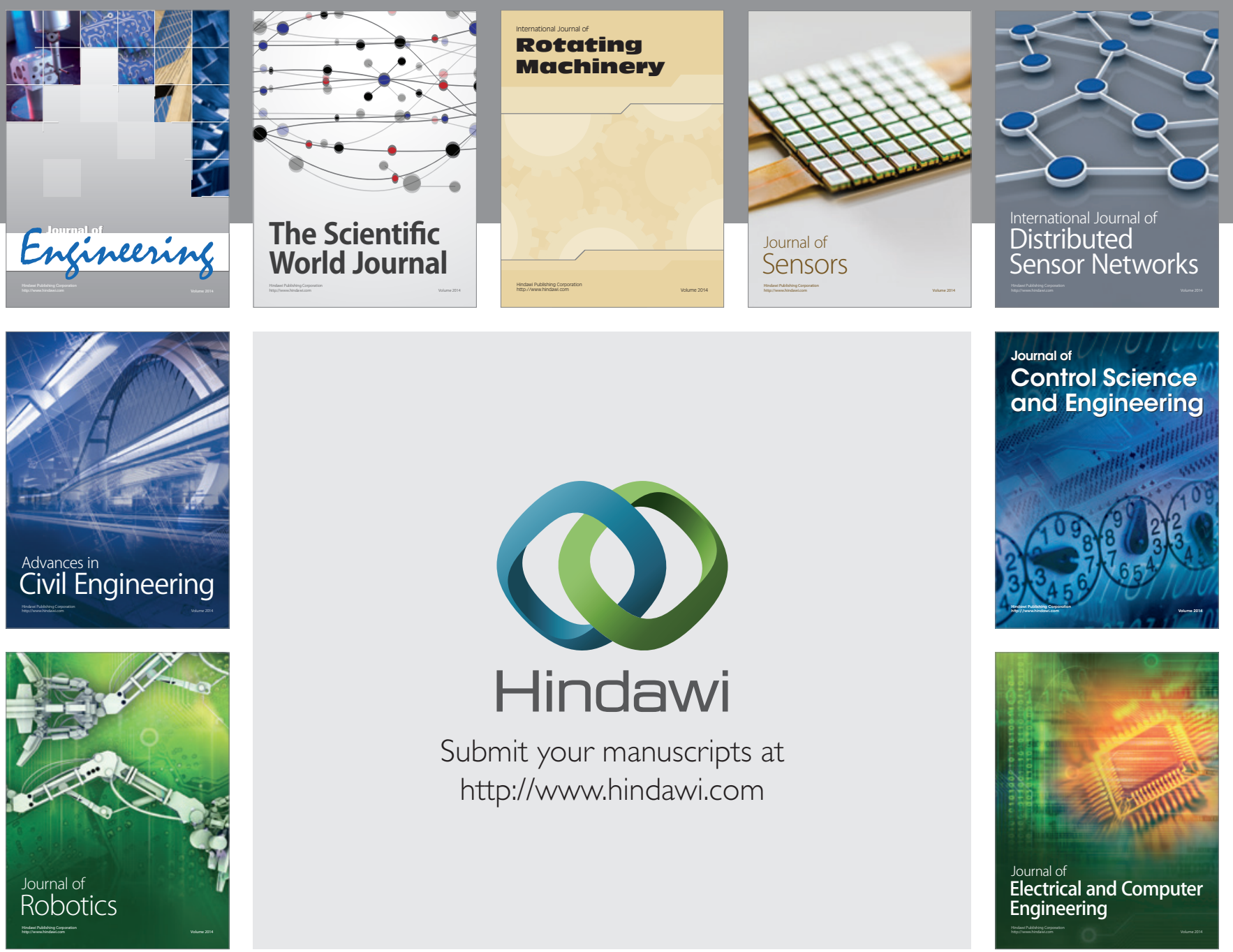

Submit your manuscripts at

http://www.hindawi.com
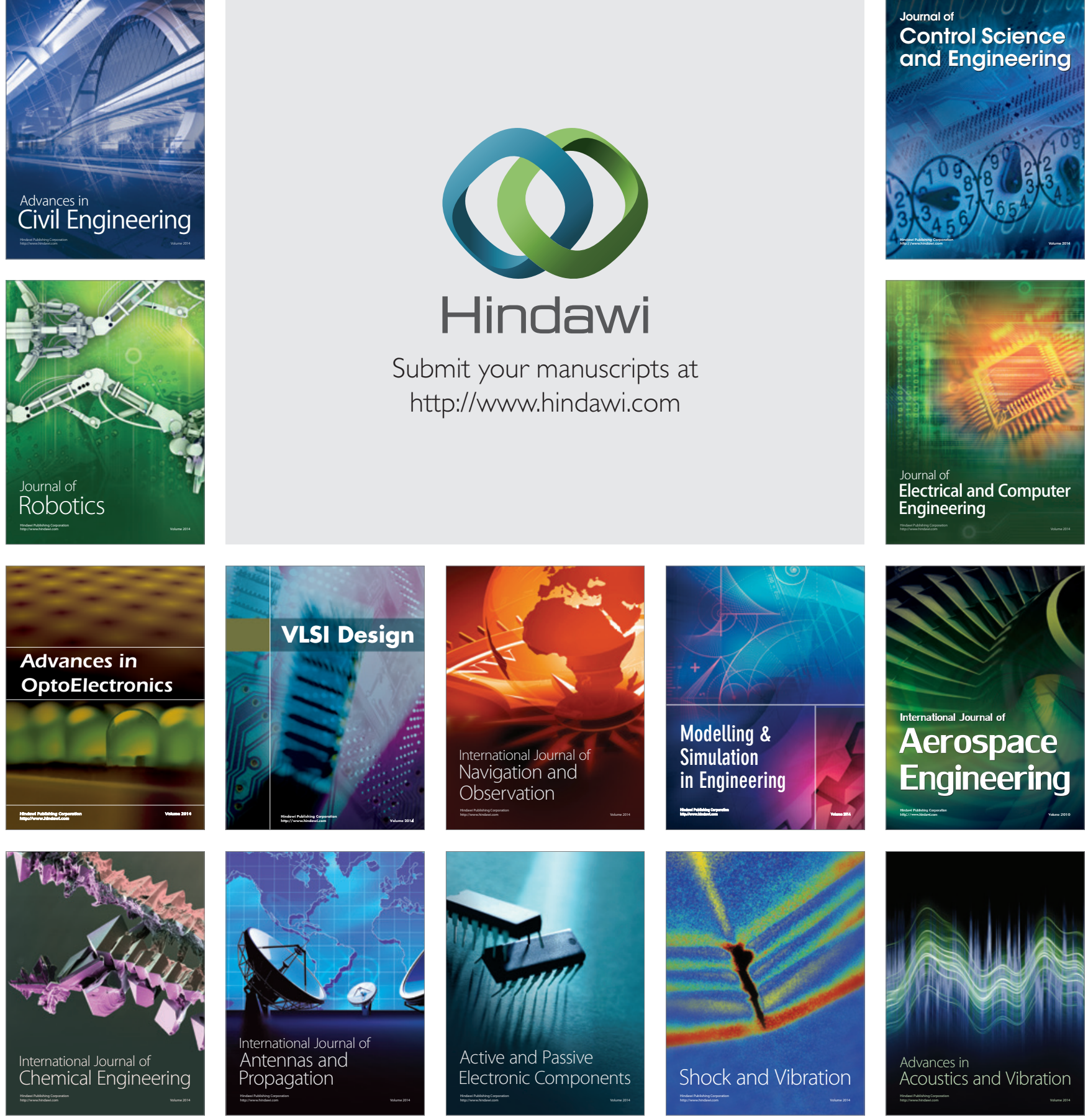